\title{
2D Satellite Image Registration Using Transform Based and Correlation Based Methods
}

\author{
Dr. H.B. Kekre \\ Senior Professor, Department of \\ Computer Science, \\ Mukesh Patel School of Technology \\ Management and Engineering \\ Mumbai, India
}

\author{
Dr. Tanuja K. Sarode \\ Asst. Professor, Computer Engineering \\ Department, \\ Thadomal Shahani college Of \\ engineering \\ Mumbai, India.
}

\author{
Ms. Ruhina B. Karani \\ Lecturer, Department of Information \\ Technology, \\ D.J. Sanghvi college of \\ engineering, \\ Mumbai, India.
}

\begin{abstract}
Image registration is the process of geometrically aligning one image to another image of the same scene taken from different viewpoints or by different sensors. It is a fundamental image processing technique and is very useful in integrating information from different sensors, finding changes in images taken at different times and inferring three-dimensional information from stereo images. Image registration can be done by using two matching method: transform based methods and correlation based methods. When image registration is done using correlation based methods like normalized cross correlation, the results are slow. They are also computationally complex and sensitive to the image intensity changes which are caused by noise and varying illumination. In this paper, an unusual form of image registration is proposed which focuses upon using various transforms for fast and accurate image registration. The data set can be a set of photographs, data from various sensors, from different times, or from different viewpoints. The applications of image registration are in the field of computer vision, medical imaging, military automatic target recognition, and in analyzing images and data from satellites. The proposed technique works on satellite images. It tries to find out area of interest by comparing the unregistered image with source image and finding the part that has highest similarity matching. The paper mainly works on the concept of seeking water or land in the stored image. The proposed technique uses different transforms like Discrete Cosine Transform, Discrete Wavelet Transform, HAAR Transform and Walsh transform to achieve accurate image registration. The paper also focuses upon using normalized cross correlation as an area based technique of image registration for the purpose of comparison. The root mean square error is used as similarity measure. Experimental results show that the proposed algorithm can successfully register the template and can also process local distortion in high-resolution satellite images.
\end{abstract}

Keywords- Discrete Cosine Transform (DCT); Discrete Wavelet Transform (DWT); HAAR Transform; Walsh transform Normalized Cross Correlation; Interst Point Area Extraction; Image Registration.

\section{INTRODUCTION}

The accelerated growth in the field of computer vision, image fusion, medical imaging, military automatic target recognition, remote cartography and astrophotography has established the need for the development of good image registration technique for the efficient retrieval of interest point area [10]. Before the development of image registration, there were difficulties in matching the images with angular distortion. As a result interest point matching result was poor [21]. Image registration is the process of transforming different sets of data into one coordinate system. The data set can be a set of photographs, data from various sensors, from different times, or from different viewpoints [1], [2], [5]. In this paper, the use of Discrete Cosine Transform (DCT), Discrete Wavelet transform (DWT), HAAR transform, Walsh transform and normalized cross correlation is investigated.

This paper presents the image registration techniques based on extracting interest point area of satellite images using the transforms mentioned above. Extracting the interest point area is achieved by computing correlation coefficient, DCT, DWT, HAAR and Walsh transforms between target unregistered image of any size and a source image of size NXN [21]. The root mean square error is used as similarity measure. Image registration is achieved by extracting interest point area and highlighting the same on the source image with the help of coordinate extraction.

The first section of this paper contains a brief review of previous relevant work. In the second section, the new algorithm is introduced in detail. Next, we present some experimental results using satellite images. Finally, some concluding remarks are provided.

\section{LITERATURE REVIEW}

Image registration algorithms can be broadly classified into two categories according to matching method: area based methods (ABM) and feature based methods (FBM) [1], [4], [5]. In ABM algorithms, small window of pixels in the sensed image is compared statistically with window of the same size in the reference image. The most commonly used methods are cross-correlation matching and least-squares matching. The centers of the matched windows are treated as control points, which can be used to solve mapping function parameters between the reference and sensed images [2]. ABM is a classical matching method. Feature-based matching techniques do not use the gray values to describe matching entities, but use image features derived by a feature extraction algorithm [1]. The form of the description as well as the type of feature used for matching depends on the task to be solved. 
Interest-point matching is problematic and remains the subject of much research within the communities of photo grammetry, remote sensing, computer vision systems, pattern recognition, and medical image processing [2]. In remote sensing, area-based algorithms are normally suitable for open terrain areas, but the feature-based approaches can provide more accurate results in urban areas. No single technique performs well in both circumstances [4]. Both algorithms have their own unique strengths and weaknesses. Neither type of algorithm can avoid the problem of dealing with ambiguity in smooth (low texture) areas. Feature-based algorithms face the additional problem of the effect of outliers (points with no correspondences) on the results [1], [2], [5], [7].

Because of the large number of feature-based algorithms used in interest-point matching, there are many classification methods for describing these algorithms. Normally, feature based algorithms can be categorized into rigid and non-rigid (according to the transformation between images), global and local (according to the image distortions), or corrected and uncorrected (according to the image variations) [1].

In addition, most of the feature-based algorithms search for correspondences and also address the refinement of a transformation function. Therefore, feature-based algorithms can also be grouped into three additional categories [3]. They either solve the correspondence only, solve the transformation only, or solve both the correspondence and the transformation. Although numerous feature-based algorithms have been developed, there is no general algorithm which is suitable for a variety of different applications. Every method must take into account the specific geometric image deformation [6].

For non-rigid local distortions, more complicated transformations are developed. TPS was proposed initially for global transformations, but it was improved for smooth local distortions for medical image registration [1], [2], [5].

\section{METHODOLOGY}

The proposed algorithm aims upon using DCT, DWT, HAAR, WALSH transforms and normalized cross correlation for the purpose of image registration. The algorithm works by dividing the source image into overlapping blocks of predefined size having some horizontal and vertical overlap. The interest point area extraction is achieved by finding DCT, HAAR and WALSH of unregistered target image and of each block of source image.

The minimum root mean square error criterion is used as a similarity measure. For applying DWT, first level decomposition of unregistered target image and each block of source image is taken. The similarity measure is used between unregistered image and each block of source image. The second approach used for interest point area extraction deals with computing normalized cross correlation between each block of source image and the unregistered target image.

\section{A. Correlation Based Approach for Image Registration}

This section of the paper focuses upon using normalized cross correlation to achieve image registration. Correlation based methods, sometimes called as template matching merge the feature detection step with the matching part [2].

\section{1) Normalized Cross Correlation Approach}

Windows of predefined size or even entire images are used for the correspondence estimation during the registration step. Area-based methods put emphasis on the feature matching step rather than on their detection. No features are detected in these approaches so the first step of image registration is omitted. Area-based methods, sometimes called correlationlike methods or template matching merge the feature detection step with the matching part. These methods deal with the images without attempting to detect salient objects [2]. Correlation coefficient can be calculated using equation (1).

$$
\begin{aligned}
& \boldsymbol{r}=\frac{\boldsymbol{S y x}}{\sqrt{\boldsymbol{S y y S y x}}} \\
& \left.=\sum_{i=1}^{n}(\boldsymbol{Y i}-\sim \boldsymbol{Y})(\boldsymbol{X i}-\sim \boldsymbol{x}) /\left(\sqrt{(} \sum_{i=1}^{n}(\boldsymbol{Y i}-\sim \boldsymbol{Y})^{2} * \sum_{i=1}^{n}(\boldsymbol{X} \boldsymbol{i}-\sim \boldsymbol{X})^{2}\right)\right)
\end{aligned}
$$$$
\text { a) Algorithm }
$$

Step 1. Resize the target image template to M X N pixels.

Step 2. Partition source image into overlapping blocks of size MX N with some horizontal and vertical overlap.

Step 3. Compute the correlation coefficient between each block of source image and that of target image template.

Step 4. Extract the region having maximum correlation from source image.

Step 5. Set the threshold by trial and error.

Step 6. Extract the coordinates of all blocks correlation greater than the threshold.

Step 7. Draw a region boundary enclosed by the coordinates.

- Advantages

1. Simple to implement.

2. Works best for open terrain areas.

3. Suitable for images with little distortion.

- Drawbacks

1. Slow because of computational complexity.

2. Cannot deal with smooth areas.

3. Sensitive to image intensity changes caused by noise or varying illumination.

\section{B. Transform Based Approach for Image Registration}

This section of the paper focuses upon making use of different transforms mentioned below to achieve image registration. The new proposed algorithm is discussed for each of these transforms along with some of the required equations for implementation of the same.

\section{1) Discrete Cosine Transform Approach}

The discrete cosine transform (DCT) is closely related to the discrete Fourier transform. It is a separable linear transformation; that is, the two-dimensional transform is equivalent to a one-dimensional DCT performed along a single dimension followed by a one-dimensional DCT in the other dimension [11]. Two dimensional DCT transform can be calculated using equation (2). 
$F(u, v)=\frac{c u}{2} * \frac{c v}{2} \sum_{y=0}^{T} \sum_{x=0}^{T} \mathrm{f}(\mathrm{x}, \mathrm{y}) \cos \left[\frac{(2 x+1) u \pi}{16}\right] \cos \left[\frac{(2 y+1) v \pi}{16}\right]$

With

$$
\begin{array}{llll}
C u=\frac{1}{\sqrt{2}} & \text { if } u=0 & C v=\frac{1}{\sqrt{2}} & \text { if } v=0 \\
C u=1 & \text { if } u>0 & C v=1 & \text { if } v>0
\end{array}
$$

The basic operation of the DCT is as follows:

- $\quad$ The input image is $\mathrm{N}$ by $\mathrm{M}$

- $\quad f(i, j)$ is the intensity of the pixel in row $i$ and column $j$

- $\mathrm{F}(\mathrm{u}, \mathrm{v})$ is the DCT coefficient in row ' $\mathrm{u}$ ' and column ' $\mathrm{v}$ ' of the DCT matrix.

a) Algorithm

Step 1. Resize the target image to M X N pixels.

Step 2. Partition source image into overlapping blocks of size $\mathrm{M} \mathrm{X} \mathrm{N}$ by taking block size manually according to the size of source image and some horizontal and vertical overlap.

Step 3. Compute the two dimensional DCT of target image and each block of source image starting from the leftmost top block.

Step 4. Calculate root mean square error between DCT of target image and each block of source image respectively.

Step 5. Compute the root mean square error.

Step 6. Set a threshold i.e. .minimum allowable error.

Step 7. The block having minimum RMSE is extracted from source image.

Step 8. Extract the coordinates of all blocks having error value less than the threshold and draw a region boundary enclosed by the coordinates.

\section{2) Haar and Walsh Transform Approach}

When $\mathrm{k}=0$, the Haar function is defined as a constant,

$$
h o(t)=\frac{1}{\sqrt{N}}
$$

When $\mathrm{k}>0$, the Haar function is defined by,

$$
\begin{aligned}
& h k(t)=\frac{1}{\sqrt{N}} *\left(2^{\frac{p}{2}}\right) \quad \frac{q-1}{2^{p}} \leq t<\frac{q-0.5}{2^{p}} \\
& h k(t)=\frac{1}{\sqrt{N}} *\left(-2^{\frac{p}{2}}\right) \quad \frac{q-0.5}{2^{p}} \leq t<\frac{q}{2^{p}} \\
& h k(t)=0 \quad \text { otherwise }
\end{aligned}
$$

Walsh transform matrix is defined using a Hadamard matrix of order N. The Walsh transform matrix row is the row of the Hadamard matrix specified by the Walsh code index, which must be an integer in the range $[0, \ldots, N-1]$. Walsh transform matrix is defined as a set of $\mathrm{N}$ rows, denoted $\mathrm{W}_{\mathrm{j}}$, for $\mathrm{j}=0,1 \ldots, \mathrm{N}-1$, which has the following properties:

- $\quad \mathrm{W}_{\mathrm{j}}$ takes on the values +1 and -1 .

- $W_{j}[0]=1$ for all $j$.

- $\quad W j \times[W k]^{T}=0$ for $\mathrm{j} \neq \mathrm{k}$ and $\mathrm{Wj} \times[\mathrm{Wk}]^{\mathrm{T}}=\mathrm{N}$ for $\mathrm{j}=\mathrm{k}$

- $\quad W_{j}$ has exactly $\mathrm{j}$ zero crossings, for $\mathrm{j}=0,1, \ldots, \mathrm{N}-1$.

- $\quad$ Each row $\mathrm{W}_{\mathrm{j}}$ is either even or odd with respect to its midpoint

\section{a) Algorithm}

Step 1. Resize the target image to M X N pixels.

Step 2. Partition source image into overlapping blocks of size $\mathrm{M} \mathrm{X} \mathrm{N}$ by taking block size manually according to the size of source image and some horizontal and vertical overlap.

Step 3. Design HAAR/WALSH kernel of size MXN.

Step 4. Apply the kernel on each block as well as on target image individually.

Step 5. Compute the average of HAAR/WALSH result.

Step 6. Calculate root mean square error between result of target image and each block.

Step 7. The block having minimum RMSE is extracted from source image.

Step 8. Extract the coordinates of all blocks having error value less than the threshold and draw a region boundary enclosed by the coordinates.

\section{3) Discrete Wavelet Transform Approach}

Wavelet analysis is similar to Fourier analysis which allows a target function over an interval to be represented in terms of an orthonormal function.

Figure 1 shows the single level decomposition diagram using discrete wavelet transfrom.

\begin{tabular}{|c|c|}
\hline LL & HL \\
\hline LH & HH \\
\hline
\end{tabular}

Figure 1. Single level decomposition using descrete wavelet transform

\section{a) Algorithm}

Step 1. Resize the target image to M X N pixels.

Step 2. Partition source image into overlapping blocks of size $\mathrm{M} \mathrm{X} \mathrm{N}$ by taking block size manually according to the size of source image and some horizontal and vertical overlap.

Step 3. Perform single level decomposition of each block and that of target image. 
Step 4. Calculate root mean square error between result of target image and each block

Step 5. The block having minimum RMSE is extracted from source image.

Step 6. Extract the coordinates of all blocks having error value less than the threshold and draw a region boundary enclosed by the coordinates.
1. Faster than area based methods.

2. Provides good result.

3. Works best for urban areas.

- Drawbacks

1. Features cannot be exactly matched because of noise.

2. Involves human intervention for manually selecting control points.

\section{- Advantages}

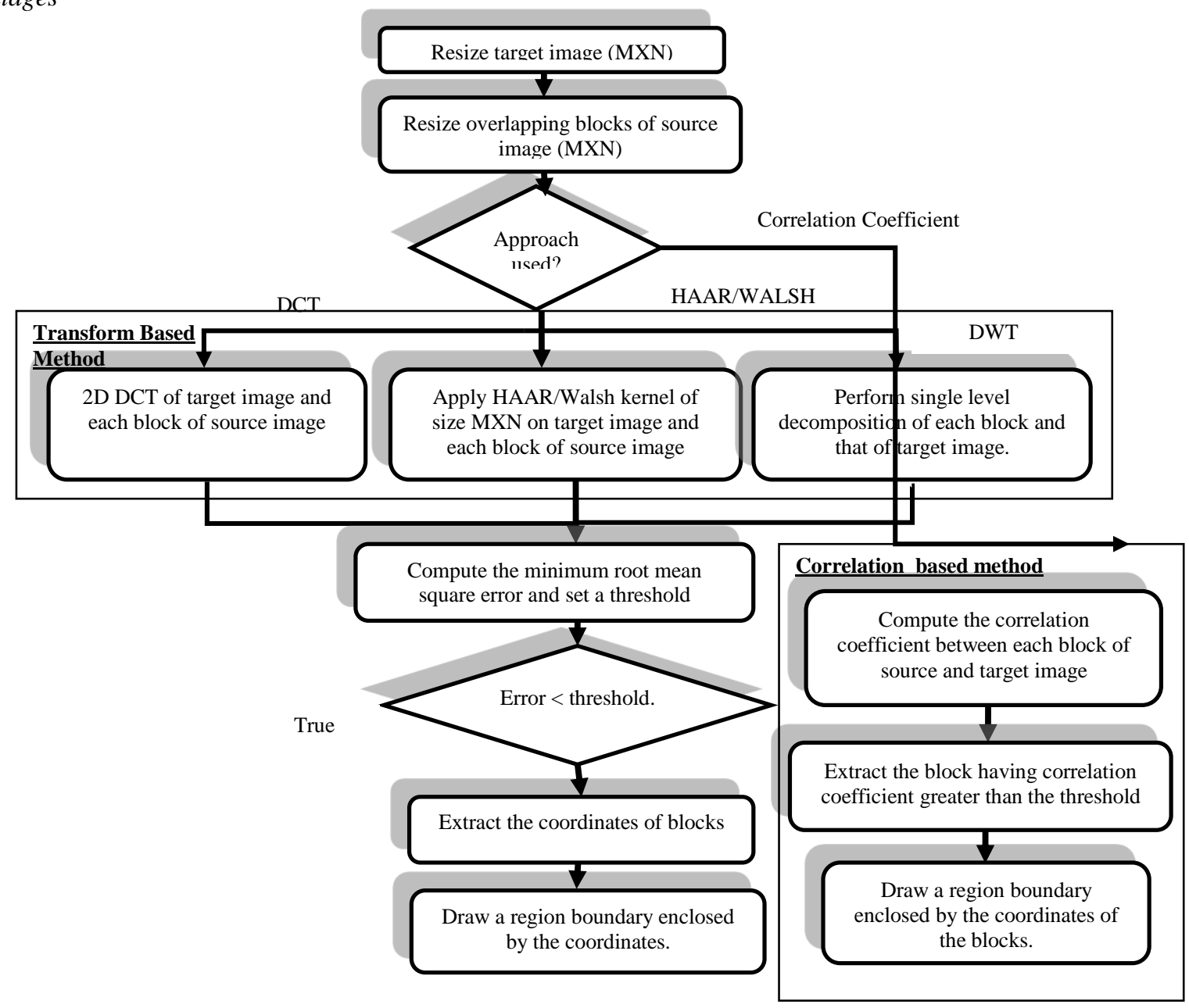

Figure 2. Flowchart for complete implementation

Figure 2 shows the complete implementation scheme of proposed technique. Initially the target image template is resized to the predefined size MXN and the stored source image is divided into overlapping blocks of same size as that of template by providing some horizontal and vertical overlap. Based on the approach used for image registration (i.e. transform based approach and correlation based approach), the corresponding algorithm mentioned for that is applied. The transform based approach applies DCT, HAAR, WALSH and wavelet transforms on the resized target image template and each block of source image. The wavelet transform uses first level decomposition technique for the same. The root mean square error is computed as a similarity measure between transformation result of target image and each block of source image. Threshold is established for minimum root mean square values. The coordinates of the corresponding blocks of source image satisfying the threshold criterion (i.e. error<threshold) are extracted and a region boundary is drawn connecting these coordinates. Correlation based approach tries to compute normalized cross correlation between the resized target image and each block of source image. The maximum value of correlation coefficient is 1 . The threshold is established for computed correlation coefficient. The coordinates of the block of source image for which correlation coefficient is greater than the threshold value are extracted and the region boundary is drawn connecting these coordinate pixels. 


\section{RESUlts AND Discussions}

The implementation of the proposed algorithm is done in MATLAB R2009a using a computer with Intel Core2 Duo Processor $(2.20 \mathrm{GHz})$ and $2 \mathrm{~GB}$ RAM.

The proposed technique works on the idea of applying image registration algorithm explained in the paper on satellite images. To achieve these results, the algorithm is executed with various satellite images of different sizes. The transform based and correlation based approach is tested on each of them. The results obtained are shown in the following figures.

\section{A. Results Obtained For Satellite Image}

This section of the paper focuses on satellite images having land and water regions. The technique takes any water image template as an input and a source satellite image is stored in a database. The goal of the technique is to find out where exaclty the water lies in the source satellite image by applying image registration.

Figure 3 shows a satellite image on which image registration has to be performed. Figure 4 shows unregistered water image temaplate taken randomly from any satellite image. Results included are obtained both by applying correlation based method as well as transform based methods. Figure 5 shows the result of image registration using normalized cross correlation approach from which it is clear that correlation based method does not detect entire water region from the source image.

Figure 6 shows the result of applying transform based approach. It shows that all the transforms i.e. DCT, HAAR, WALSH and WAVELET transfroms produce the same results. Figure 6 also shows that the result obtained by transform based approach cover most of the water region except some. Results obtained in this section imply that transform based approach performs better than correlation based approach for image registration.

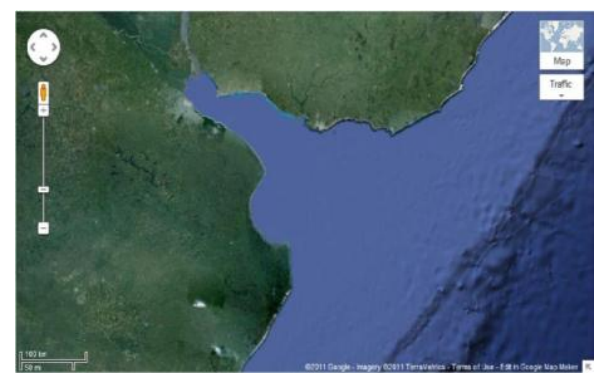

Figure 3. Source Image

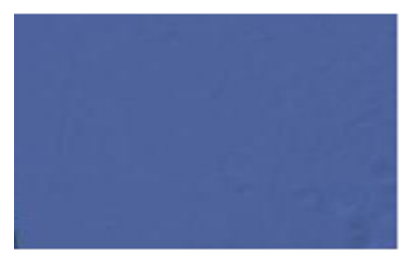

Figure 4. Unregistered Image of Water

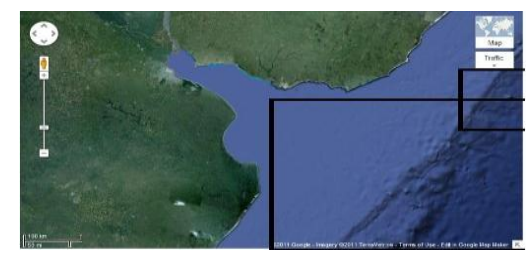

Figure 5. Registered image using normalized cross correlation

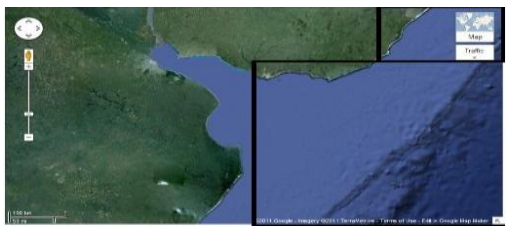

Figure 6. Registered image using DCT/DWT/WALSH/HAAR transforms

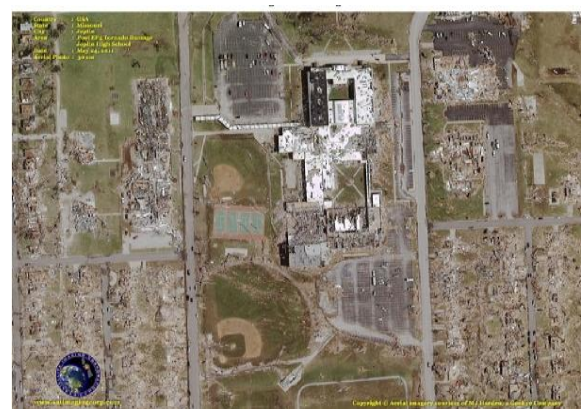

Figure 7. Source image taken from different angle

\section{B. Results Obtained For Angularly Distorted Images}

This section of the paper focuses on satellite images captured from different camera angles or by different viewpoints. The goal of this section is to register the image template taken out from the image taken from one angle to the image taken from some different angle thus satisfying the basic definition of image registration explained in section I. Figure 7 shows image of some region captured from one angle whereas Figure 8 shows the same image captured from different angle. The image registration technique mentioned in the paper works by extracting image template of size MXN as shown in figure 9 and figure 10 from the source image taken from different angle.

The source image 1 is then divided into overlapping blocks of same size as the template by providing some horizontal and vertical overlap. Both the techniques (i.e. Correlation Based Approach and Transform Based Approach) are applied on the template and each block of source image. Figure 11 shows the result obtained by applying both the methods for registration of the image template shown in figure 9.

Figure 12 shows the result obtained by applying both the methods for registration of the image template shown in figure 10. Experimental results imply that both the technique produce same result. Both the techniques correctly outline the interest point area. Experimental results also show that Correlation based approach consumes more time to produce the result as compared to Transform based approach. 


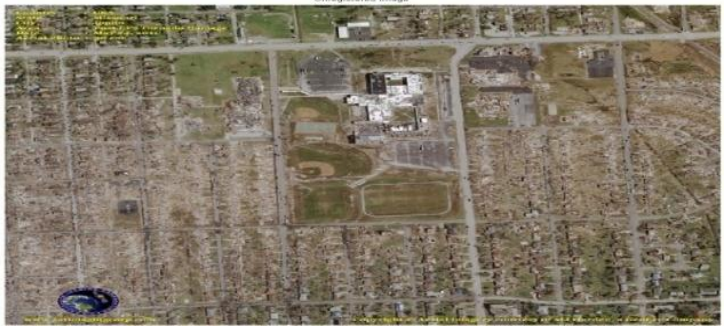

Figure 8. Source image 1

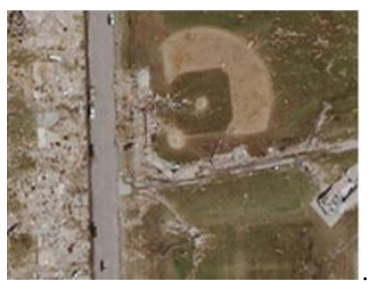

Figure 8. Unregistered image template 1

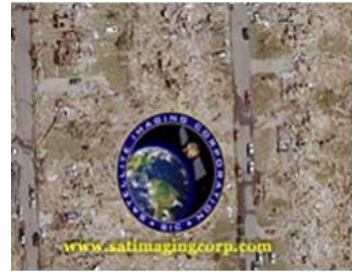

Figure 9. Unregistered image template 2

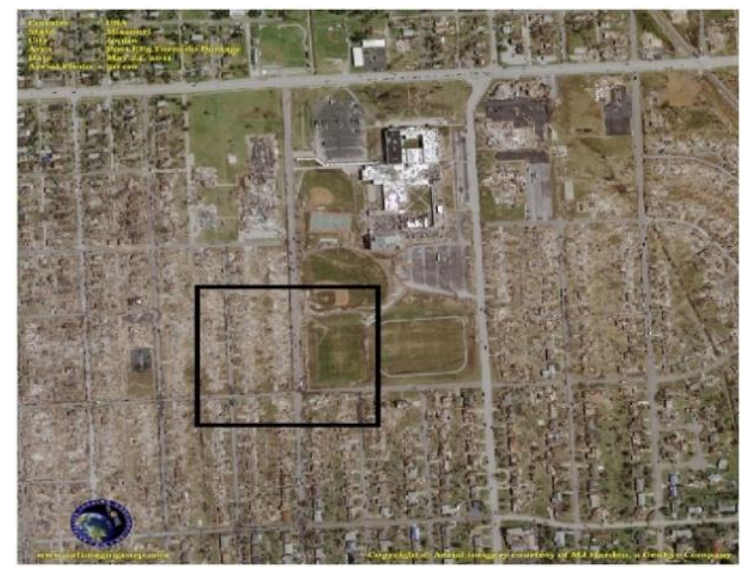

Figure 10. Registered image 1 using correlation based and transfrom based methods

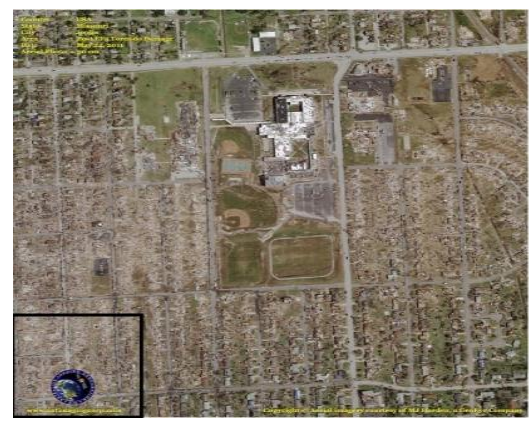

Figure 11. Registered image 2 using correlation based and transfrom based methods

\section{CONCLUSION}

Image registration plays an important role in the alignment of two or more images of the same scene. In this paper, a simple but effective algorithm of Image Registration is presented which uses transform based approach as well as area based approach. The algorithm works with the idea of dividing the source image into overlapping blocks of size same as that of unregistered target image.

The image registration method of this paper is for the field of satellite imaging. Image registration is achieved by extracting the coordinates of blocks satisfying the criteria of threshold value thus making entire procedure automatic for interest point area detection. This paper also takes angularly distorted images into consideration. It tries to locate the exact position of given template of the image taken from different angle or by different viewpoint thus achieving basic definition of image registration.

Experimental results on various images in image registration shows that the interest point area computed using transform based approach provides more accurate results as compared to the registration performed using normalized cross correlation.

\section{REFERENCES}

[1] Xiong and Yun Zhang "A Novel Interest-Point-Matching Algorithm for High-Resolution Satellite Images" ieee transactions on geoscience and remote sensing, vol. 47, no. 12, december 2009.

[2] Gang Hong, Yun Zhang "Combination of Feature-based and Area- based Image Registration Technique for High Resolution Remote Sensing Image" ieee international conference on Geoscience and Remote Sensing Symposium, 2007.

[3] Ronald W. K. So and Albert C. S. Chung "Multi-level non-rigid image registration using graph-cuts" ieee international conference on Acoustics, Speech and Signal Processing, 2009.

[4] M. Auer, P. Regitnig, and G. A. Holzapfel, "An automatic non rigid registration for stained histological sections," IEEE Trans. Image Process.,vol. 14, no. 4, pp. 475-486, Apr. 2005.

[5] Goshtasby, A., "Piecewise linear mapping functions for image registration, Pattern Recognition", vol.19, no.6, pp.459-466,1986.

[6] Dongjin Kwon, "Rolled Fingerprint Construction Using MRF-Based Nonrigid Image Registration", ieee transactions on image processing, vol. 19, no. 12, december 2010

[7] Boffy, Y. Tsin, and Y. Genc, "Real-time feature matching using adaptive and spatially distributed classification trees," Brit. Mach. Vis. Conf., Jul. 2006.

[8] Ming-Sui Lee, Meiyin Shen, Akio Yoneyama and C. -C. Jay Kuo "DCTDomain Image Registration Techniques for Compressed Video" ieee international conference on circuits and systems, 2005.

[9] L. G. Brown, "A survey of image registration techniques," ACM Comput.Surv., vol. 24, no. 4, pp. 325-376, Dec. 1992.

[10] Dr. H.B.Kekre, Sudeep D. Thepade, Akshay M aloo, "Image Retrieval using Fractional Coefficients of Transformed Image using DCT and Walsh Transform", International Journal of Engineering Science and Technology, Vol. 2(4), pp .362-371, 2010.

[11] H. Chui and A. Rangarajan, "A new point matching algorithm for nonrigid registration," Comput. Vis. Image Underst., vol. 89, no. 2/3, pp. 114-141, Feb. 2003.

[12] C. Harris and M. Stephens, "A combined corner and edge detector," in Proc. Alvey Vis. Conf., 1988, pp. 147-151.

[13] G. K. Wallace, "Overview of the JPEG still Image Compression standard," SPIE 1244 (1990) 220-233.

[14] J. Rexilius, S. K. Warfield, C. R. G. Guttmann, X. Wei, R. Benson,L. Wolfson, M. Shenton, H. Handels, and R. Kikinis,"A novel 
nonrigidregistration algorithm and applications," in Proc.MICCAI, W. Niessen and M. Viergever, Eds., 2001, vol. 2208, pp. 923-931.

[15] J. Williams and M. Bennamoun, "Simultaneous registration of multiple corresponding point sets," Comput. Vis. Image Underst., vol. 81, no. 1, pp. 117-142, Jan. 2001

[16] Sang-M i Lee, Hee_Jung Bae, and Sung-Hwan Jung, "Efficient ContentBased Image Retrieval M ethods Using Color and Texture", ETRI Journal 20 (1998) 272-283.

[17] Sahil Suri and Peter Reinartz, "Mutual-Information-Based Registration of TerraSAR-X and Ikonos Imagery in Urban Areas" ieee transactions on geoscience and remote sensing, vol. 48, no. 2, february 2010

[18] Mingchao Sun, Bao Zhang, linghong Liu, Yongyang Wang and Quan Yang," The Registration of Aerial Infrared and Visible Images" International Coriference on Educational and Information Technology 2010.

[19] L. Zagorchev, and A. Goshtasby, "A comparative study of transformation functions for nonrigid image registration," IEEE Transactions on Image Processing, vol.15, pp. 529-538, 2006.

[20] Ruhina Karani, Dr. Tanuja K. Sarode, "Image Registration using Discrete Cosine Transform and Normalized Cross Correlation", International Journal of Computer Application, ISBN No- 973-93$80864-48-5,2012$

\section{AUTHORS PROFILE}

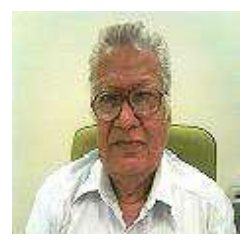

Dr. H. B. Kekre has received B.E. (Hons.) in Telecomm. Engineering. from Jabalpur University in 1958, M.Tech (Industrial Electronics) from IIT Bombay in 1960, M.S.Engg. (Electrical Engg.) from University of Ottawa in 1965 and Ph.D. (System Identification) from IIT Bombay in $1970 \mathrm{He}$ has worked as Faculty of Electrical Engg. and then HOD Computer Science and Engg. at IIT Bombay. For 13 years he was working as a professor and head in the Department of Computer Engg. at Thadomal Shahani Engineering. College, Mumbai. Now he is Senior Professor at MPSTME, SVKM's NMIMS University. He has guided 17 Ph.Ds, more than 100 M.E./M.Tech and several B.E./B.Tech projects.
His areas of interest are Digital Signal processing, Image Processing and Computer Networking. He has more than 350 papers in National / International Conferences and Journals to his credit. He was Senior Member of IEEE. Presently He is Fellow of IETE and Life Member of ISTE Recently ten students working under his guidance have received best paper awards and two have been conferred Ph.D. degree of SVKM's NMIMS University.Currently 10 research scholars are pursuing Ph.D. program under his guidance.

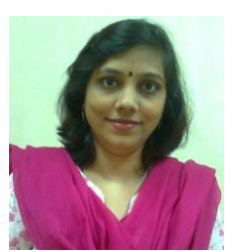

Dr. Tanuja K. Sarode has Received Bsc.(Mathematics) from Mumbai University in 1996, Bsc.Tech.(Computer Technology) from Mumbai University in 1999, M.E. (Computer Engineering) degree from Mumbai University in 2004, Ph.D. from Mukesh Patel School of Technology, Management and Engineering, SVKM"s NMIMS University, Vile-Parle (W), Mumbai, INDIA. She has more than 11 years of experience in teaching. Currently working as Assistant Professor in Dept. of Computer Engineering at Thadomal Shahani Engineering College, Mumbai. She is life member of IETE, member of International Association of Engineers (IAENG) and International Association of Computer Science and Information Technology (IACSIT), Singapore. Her areas of interest are Image Processing, Signal Processing and Computer Graphics. She has more than 100 papers in National /International Conferences/journal to her credit.

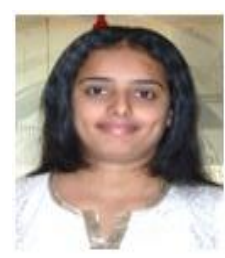

Ms. Ruhina B. Karani has received B.E. (Computer Engineering) from Mumbai University in 2007. Currently pursuing her M.E. (Computer Engineering) from Thadomal Shahani Engineering College, Mumbai. She has 4 years of experience in teaching. She was working as a lecturer in K.J. Somaiya College of engineering and is currently working as a lecturer in D.J. Sanghvi college of engineering, Mumbai. She is a life time member of ISTE. Her areas of interest are Image processing, Microprocessor and microcontroller. She has guided two B.E. projects. She has one research paper in international journal to her credit. 